\title{
Savants sous l'Occupation. Enquête sur la vie scientifique française entre 1940 et 1944
}

Nicolas Chevassus-au-Louis. Paris, Éditions du Seuil, collection « Science ouverte », 2004

\section{Catherine Nicault}

\section{(2) OpenEdition}

\section{Journals}

Édition électronique

URL : https://journals.openedition.org/histoire-cnrs/438

DOI : $10.4000 /$ histoire-cnrs.438

ISSN : 1955-2408

Éditeur

CNRS Éditions

Édition imprimée

Date de publication : 3 mai 2005

ISBN : 978-2-271-06250-5

ISSN : $1298-9800$

Référence électronique

Catherine Nicault, « Savants sous I'Occupation. Enquête sur la vie scientifique française entre 1940 et 1944 », La revue pour l'histoire du CNRS [En ligne], 12 | 2005, mis en ligne le 07 mars 2006, consulté le 20 mai 2021. URL : http://journals.openedition.org/histoire-cnrs/438 ; DOI : https://doi.org/10.4000/ histoire-cnrs.438

Ce document a été généré automatiquement le 20 mai 2021.

Comité pour l'histoire du CNRS 


\section{Savants sous l'Occupation. Enquête sur la vie scientifique française entre 1940 et 1944}

Nicolas Chevassus-au-Louis. Paris, Éditions du Seuil, collection « Science ouverte », 2004

\section{Catherine Nicault}

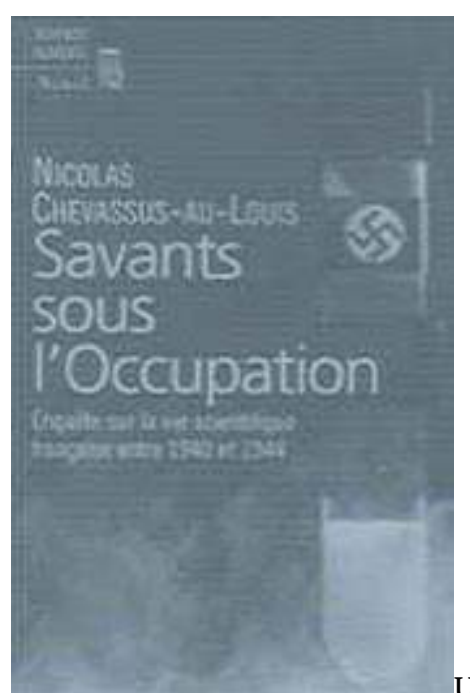

Une fois n'est pas coutume : cette synthèse rédigée d'une plume alerte devrait réunir à la fois les suffrages des historiens professionnels et ceux du " grand public », tout au moins des curieux de la chose scientifique et de la période de l'Occupation. Le journaliste Nicolas Chevassus-au-Louis a su en effet peindre le sort des chercheurs et des institutions scientifiques pendant l'Occupation - l'histoire de la recherche elle-même n'est évoquée qu'épisodiquement - avec une rigueur de méthode et un talent d'exposition faits pour contenter les premiers sans rebuter les autres.

1 De cette réussite, qui a déjà valu à l'ouvrage une flatteuse couverture de presse, quelle est donc la recette? D'abord, et il faut le souligner avec force, une enquête documentaire de première qualité : avant de prétendre traiter de cette question 
délicate, encore relativement peu traitée dans l'historiographie et parfois sujette à controverses, l'auteur a absorbé avec intelligence à peu près toute la littérature historique disponible, y compris les travaux universitaires inédits, de la thèse de doctorat au simple mémoire de maîtrise, interrogé des témoins et procédé à des recherches ciblées dans les archives. D'où un texte parfaitement informé, dépourvu de ces irritants à peu près qui disqualifient trop souvent la (mauvaise) vulgarisation aux yeux des spécialistes.

2 De cette solide collecte de l'information témoigne d'ailleurs un réseau nourri de références qui parvient à ne pas nuire à l'agrément de la lecture. C'est que l'auteur a pris le parti astucieux de moduler la présence de l'appareil critique en fonction des différentes composantes de l'ouvrage. Seul le premier gros chapitre, «Scientifiques des années noires", qui dresse un tableau des avatars de la vie des chercheurs et des laboratoires pendant la guerre, ainsi que les dix pages de la conclusion, «Scientifiques ou intellectuels", comportent un appareil de notes infrapaginales. Le prologue en revanche - consacré en fait au chimiste Bernard Halpern -, ainsi que les douze récits à caractère biographique qui servent ensuite à illustrer et approfondir les aspects marquants de la période, en sont dépourvus ; pour autant le lecteur est éclairé sur les ingrédients entrant dans la composition de ces récits par la mention circonstanciée à la fin de chacun d'eux des sources utilisées ainsi que, le cas échéant, des interprétations faisant débat ou encore des raisons du choix opéré par l'auteur entre les diverses versions possibles de certains épisodes.

3 Le pari de l'exposition consiste en effet à avoir limité la part faite à la synthèse aux soixante pages du premier chapitre, puis à les avoir complétées et animées en contrepoint par douze portraits-récits soigneusement choisis, d'une dizaine de pages chacun. Il en résulte, non pas le patchwork que l'on pourrait craindre, mais une tapisserie étonnamment cohérente qui retient l'attention du lecteur par la variété des approches proposées.

Le tableau initial brosse donc une image d'ensemble de la vie scientifique pendant les années noires: l'impact de la débâcle de juin 1940 qui désorganise la recherche et disperse les chercheurs; les conditions difficiles de la reprise de l'activité scientifique $\mathrm{du}$ fait des pénuries, de la disparition des supports de publication comme de la restriction des communications; le manque d'intérêt des Allemands pour la science française en dehors du domaine de la physique nucléaire ; la volonté de modernisation scientifique de Vichy - qui conserve finalement le CNRS, création du Front populaire, mais laisse les ministères créer leurs propres organismes de recherches spécialisés mais aussi son souci, là comme ailleurs, d'«aryaniser » le milieu scientifique; la possibilité jusqu'en 1941 pour les scientifiques renommées de choisir «légalement » l'exil (le rôle du Service des œuvres du Quai d'Orsay aurait mérité du reste d'être davantage souligné); les options politiques divergentes du monde savant partagé comme tous les autres milieux entre résistance, collaboration (rare en fait comparé aux écrivains, en raison surtout du faible niveau des sollicitations allemandes) et surtout accommodation; l'épuration enfin, bien plus légère dans les institutions de recherche que dans les universités, et la réorganisation de la science française après la Libération.

5 Sur cette toile de fond, les récits plus anecdotiques, toujours traités avec pondération et doigté, viennent broder du détail et de la vie, c'est-à-dire de la complexité. Ils sont évidemment l'occasion de traiter des différends entre Lucien Febvre et Marc Bloch, des cas de Frédéric Joliot-Curie, du docteur Alexis Carrel et de sa Fondation française pour 
l'étude des problèmes humains, du groupe Bourbaki ou encore de Louis Rapkine, maître d'œuvre du bureau scientifique de la France libre à New York, tous objets de travaux récents. D'autres sont consacrés à des personnalités moins étudiées : par exemple Charles Jacob, directeur du CNRS de Vichy, dont le portrait est sans doute trop indulgent à en croire une étude fondée sur les archives de l'Institut que notre revue publiera prochainement, l'antisémite frénétique que fut l'« ethnoraciste» Georges Montandon, Raymond Croland, lumineuse figure de résistant, ou les chimistes Jean Gérard, Gaston Ramon et Ernest Fourneau ainsi que le physicien et ingénieur Georges Claude, accusés à un degré ou à un autre de collaboration après la guerre.

Bref un livre utile et plaisant à la fois qui montre notamment que les laboratoires n'ont pas été touchés autant qu'on aurait pu le croire par les malheurs du temps grâce au soutien donné à la modernisation scientifique par Vichy et à l'intérêt très limité de l'occupant pour la recherche française. Quant aux chercheurs, leur comportement ne diffère guère de celui de leurs compatriotes pendant les années noires : pour un certain nombre engagés dans la résistance intérieure et extérieure ou, à l'inverse, dans la collaboration, la plupart, et non des moindres, se sont "accommodés", ne prenant leurs distances avec le nouveau régime qu'à partir de 1942.

\section{AUTEUR}

CATHERINE NICAULT

Professeure à l'université de Reims 